\title{
Review of: "Color attributes, betacyanin, and carotenoid profiles, bioactive components, and radical quenching capacity in selected Amaranthus gangeticus leafy vegetables"
}

Vesna Tumbas Šaponjac ${ }^{1}$

1 University of Novi Sad

Potential competing interests: The author(s) declared that no potential competing interests exist.

The manuscript "Color attributes, betacyanin, and carotenoid profiles, bioactive components, and radical quenching capacity in selected Amaranthus gangeticus leafy vegetables" contains interesting information about the phytopigments, nutraceuticals, and antioxidants present in four high-yielding A. gangeticus accessions.

The work was carried out in a competent fashion. Methods for determination of the color attributes, betacyanins, and carotenoid profiles, and antioxidants potentials are technically and methodologically sound.

The aim and methods of the research are well described and conducted in this paper, giving the satisfactory results. The conclusions are adequately supported by data.

I think that the research reported in this paper is relevant and likely to be of great interest to readers of your reputable Journal and therefore I recommend the Editorial board to accept it for publication in the present form. 